\title{
Particle-Hole Symmetry and the Effect of Disorder on the Mott-Hubbard Insulator
}

\author{
P. J. H. Denteneer \\ Lorentz Institute, Leiden University, P.O. Box 9506, 2300 RA Leiden, The Netherlands \\ R. T. Scalettar \\ Physics Department, University of California, 1 Shields Avenue, Davis, California 95616 \\ N. Trivedi \\ Department of Theoretical Physics, Tata Institute of Fundamental Research, Homi Bhabha Road, Mumbai 400005, India
}

(Received 5 June 2001; published 18 September 2001)

\begin{abstract}
The understanding of the interplay of electron correlations and randomness in solids is enhanced by demonstrating that particle-hole $(p-h)$ symmetry plays a crucial role in determining the effects of disorder on the transport and thermodynamic properties of the half-filled Hubbard Hamiltonian. We show that the low-temperature conductivity decreases with increasing disorder when $p$ - $h$ symmetry is preserved, and shows the opposite behavior, i.e., conductivity increases with increasing disorder, when $p$ - $h$ symmetry is broken. The Mott insulating gap is insensitive to weak disorder when there is $p$ - $h$ symmetry, whereas in its absence the gap diminishes with increasing disorder.
\end{abstract}

Introduction.-The interplay of disorder and interactions is at the heart of many interesting and unexplained phenomena in condensed matter physics. For example, the effects of disorder and interactions in two-dimensional (2D) electronic systems acting separately lead to insulating behavior of the Anderson and Mott kinds, respectively. However, experimental findings on silicon metal-oxidesemiconductor field-effect transistors (MOSFETs) show the occurrence of a conducting phase [1], which is the result of the combined importance of randomness and interactions [2,3]. Other examples of situations in which both disorder and interactions are crucial, yet incompletely understood, include the formation of local moments and the behavior of the susceptibility in doped semiconductors [4], the superconductor-insulator transition and universal conductivity in thin metallic films [5,6], and the pinning of flux lines in type-II superconductors [7].

In recent years, it has become increasingly clear that for noninteracting electrons the presence or absence of certain symmetries is crucial in determining the effect of disorder on both transport and thermodynamic properties, as well as critical properties of the localization transition [8]. Recent examples where symmetry considerations are important are given in the context of quantum wires [9] and disordered superconductors $[10,11]$, where chiral, time-reversal, and spin-rotation symmetries play an important role.

In this paper, we examine the effect of different types of disorder on both the dynamic and equilibrium thermodynamics of the 2D Hubbard model in the vicinity of half filling, electron density $\langle n\rangle=1$. Our results suggest that the presence or absence of particle-hole symmetry determines the effect of randomness on the conductivity and the Mott gap.

The model and computational approach. - We consider the following 2D Hubbard Hamiltonian,

$$
\begin{aligned}
H= & -\sum_{\langle\mathbf{i} \mathbf{j}\rangle, \sigma} t_{\mathbf{i j}} c_{\mathbf{i} \sigma}^{\dagger} c_{\mathbf{j} \sigma}-\sum_{\langle\langle\mathbf{i} \mathbf{k}\rangle, \sigma} t_{\mathbf{i k}}^{\prime} c_{\mathbf{i} \sigma}^{\dagger} c_{\mathbf{k} \sigma} \\
& +U \sum_{\mathbf{j}}\left(n_{\mathbf{j} \uparrow}-\frac{1}{2}\right)\left(n_{\mathbf{j} \downarrow}-\frac{1}{2}\right)-\sum_{\mathbf{j}, \sigma} \mu_{\mathbf{j}} n_{\mathbf{j} \sigma} .
\end{aligned}
$$

Here $t_{\mathbf{i j}}$ is a bond-dependent hopping matrix element on nearest-neighbor sites $\langle\mathbf{i} \mathbf{j}\rangle, t_{\mathbf{i k}}^{\prime}$ is a bond-dependent hopping matrix element on next-nearest-neighbor sites $\langle\langle\mathbf{i k}\rangle\rangle$, $U$ is an on-site repulsion, and $\mu_{\mathbf{j}}$ is a site-dependent chemical potential. We choose $P\left(t_{\mathbf{i j}}\right)=1 / \Delta_{t}$ for $t_{\mathbf{i j}} \in$ $\left[t-\Delta_{t} / 2, t+\Delta_{t} / 2\right]$, and zero otherwise, with $t=1$ to set our scale of energy. Similarly, $P\left(t_{\mathbf{i k}}^{\prime}\right)=1 / \Delta_{t}^{\prime}$ for $t_{\mathbf{i k}}^{\prime} \in$ $\left[t^{\prime}-\Delta_{t}^{\prime} / 2, t^{\prime}+\Delta_{t}^{\prime} / 2\right]$, and $P\left(\mu_{\mathbf{j}}\right)=1 / \Delta_{\mu}$ for $\mu_{\mathbf{j}} \in$ $\left[-\Delta_{\mu} / 2,+\Delta_{\mu} / 2\right]$, so that the various $\Delta$ measure the disorder strength. We will focus on half filling where the effects of interactions are most prominent, as evidenced by the formation of antiferromagnetic correlations and a Mott-Hubbard charge gap at low temperatures.

Our computational technique is determinant quantum Monte Carlo (QMC) [12], an approach which allows us to study much larger numbers of particles than those explored with exact diagonalization (and the two-electron problem) $[13,14]$. In this method the electron-electron interactions are replaced by a space- and imaginary-time-dependent Hubbard-Stratonovich field. The integral over possible field configurations, which exactly retains the interactions in the problem, is done stochastically and allows us to calculate static and dynamic (Matsubara time) correlation functions at a fixed temperature $T$. All our results are for lattices of $8 \times 8$ spatial sites and coupling $U=4 t$. We averaged over up to 16 disorder realizations; the error bars indicate the statistical fluctuations from disorder sampling.

The disordered Hubbard model in Eq. (1) is particlehole $(p-h)$ symmetric when $t_{\mathbf{i k}}^{\prime}=\mu_{\mathbf{j}}=0$. That is, under 
the transformation $c_{\mathbf{i} \sigma}^{\dagger} \rightarrow(-1)^{\mathbf{i}} c_{\mathbf{i} \sigma}$ the Hamiltonian is unchanged, and the system is precisely half filled for all values of the parameters in $H$ and also for all $T$. Therefore, while nearest-neighbor bond and local site disorder both introduce randomness into the system, they differ fundamentally in that site disorder breaks $p$ - $h$ symmetry.

In order to reveal the effects of disorder quantitatively, we will examine the transport by evaluating the temperature-dependent dc conductivity,

$$
\sigma_{\mathrm{dc}} \simeq \frac{\beta^{2}}{\pi} \Lambda_{x x}(\mathbf{q}=0, \tau=\beta / 2)
$$

(with $\beta \equiv 1 / k_{\mathrm{B}} T$ ) as determined [15] from the currentcurrent correlation function, $\Lambda_{x x}(\mathbf{q}, \tau)=$ $\left\langle j_{x}(\mathbf{q}, \tau) j_{x}(-\mathbf{q}, 0)\right\rangle$. Here $j_{x}(\mathbf{q}, \tau)$, the $\mathbf{q}, \tau$-dependent current in the $x$ direction, is the Fourier transform of $j_{x}(\mathbf{l})=i \sum_{\sigma} t_{\mathbf{1}+\hat{x}, \mathbf{I}}\left(c_{\mathbf{1}+\hat{x}, \sigma}^{\dagger} c_{\mathbf{l} \sigma}-c_{\mathbf{1} \sigma}^{\dagger} c_{\mathbf{1}+\hat{x}, \sigma}\right)$. From the one-electron Green function as a function of imaginary time we extract the temperature-dependent density of states at the chemical potential $N(\epsilon=0)$ [16]:

$$
N(0) \simeq-\beta G(\mathbf{r}=0, \tau=\beta / 2) / \pi .
$$

These two quantities allow a clear characterization of the transport and thermodynamic properties of the system. For $\sigma_{\mathrm{dc}}$ and $N(0)$, "Trotter" errors associated with the discretization of imaginary time $\beta$ are considerably less than the fluctuations associated with Monte Carlo and disorder averaging.

Results.-First we discuss the transport properties: in Fig. 1, we exhibit the effect of nearest-neighbor hopping (bond) disorder on the conductivity. For all disorder strengths $\Delta_{t}$, at temperatures greater than a characteristic temperature $T_{*}$ related to the Mott gap, the system shows metallic behavior with $\sigma_{\mathrm{dc}}$ increasing upon lowering $T$. The conductivity turns down sharply as the temperature

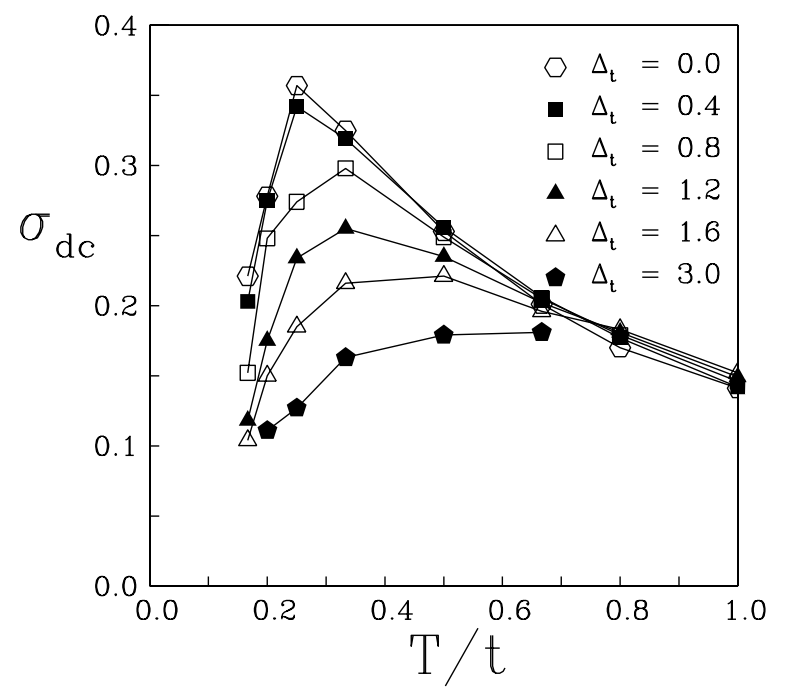

FIG. 1. The effect of particle-hole-symmetry preserving (nearest-neighbor) bond disorder in the half-filled Hubbard Hamiltonian is to decrease the conductivity $\sigma_{\mathrm{dc}}$. Data are for $U=4 t$ on a $8 \times 8$ square lattice; $\Delta_{t}$ measures the strength of the bond disorder. drops below $T_{*}$ and the system shows insulating behavior with $\sigma_{\mathrm{dc}}$ decreasing upon lowering $T$. In the case of zero randomness, the perfect nesting of the Fermi surface in 2D leads to antiferromagnetic long range order (AFLRO) in the ground state with an associated spin density wave gap for arbitrarily small $U$ evolving to a Mott gap at larger $U$. Hopping disorder reduces AFLRO via the formation of singlets on bonds with large hopping $t_{\mathbf{i j}}$ and hence large coupling $J=t_{\mathbf{i j}}^{2} / U$ and ultimately destroys it beyond $\Delta_{t} \approx 1.6 t$ [17]. The fascinating result we have found is that insulating behavior in the conductivity nevertheless persists to much larger $\Delta_{t}$. Moreover, from the shift of the maximum in Fig. 1 we deduce that the mobility gap in fact increases with increasing $\Delta_{t}$.

The situation is quite different in the case of site disorder, as shown in Fig. 2: at fixed temperature $T$, as site disorder $\Delta_{\mu}$ is turned on, the conductivity increases; i.e., the Mott insulating state is weakened [18]. At weak disorder, the conductivity drops with decreasing $T$, reflecting again the presence of the Mott insulating phase. As the disorder strength becomes large enough to neglect $U$, one would expect a similar temperature dependence arising from Anderson insulating behavior. We believe that in all cases the conductivity will ultimately turn over and go to zero at low $T$, but we are limited in these simulations to temperatures $T>W / 48$ because of the fermion sign problem. Nevertheless, the data for site disorder offer a dramatic contrast to that of bond disorder (Fig. 1) where randomness decreases the conductivity.

What is the underlying reason for the different effects of bond and site disorder on conductivity? There are several obvious differences in the effect of bond and site disorder on local and even longer range spin and charge correlations. Site disorder enhances the amount of double occupancy on the lattice, since the energy cost $U$ of double

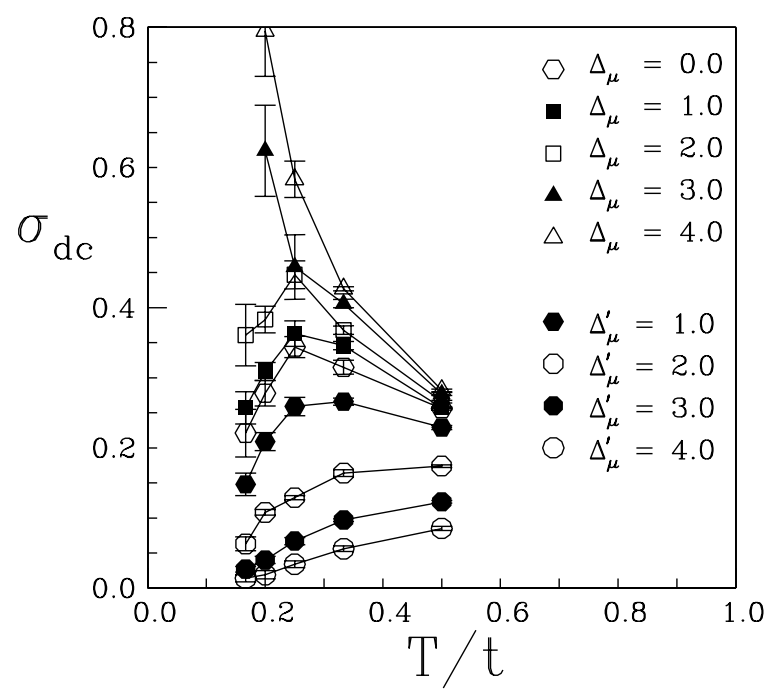

FIG. 2. Canonical site disorder (with strength $\Delta_{\mu}$ ) enhances the conductivity. Particle-hole-symmetric site disorder (with strength $\Delta_{\mu}^{\prime}$ ), as with bond disorder (Fig. 1), suppresses the conductivity. Other parameters are as in Fig. 1. 
occupancy is compensated by differences in site energies. One explanation of why site disorder increases $\sigma_{\mathrm{dc}}$ is that the concomitant increase in empty sites leads to more mobility. This destruction of local moments ultimately also leads to the end of antiferromagnetic order. Surprisingly, we find in our simulations that bond disorder has a similar diminishing effect on local moments, suggesting that the difference in the behavior of the conductivity arises from a different origin.

We argue here that particle-hole symmetry is the unifying criterion which underlies and determines the effect of disorder. As emphasized above, site and bond disorder have rather similar effects on the double occupancy. Moreover, the consequences of this effect for $\sigma_{\mathrm{dc}}$ are expected to become visible only above a threshold value of disorder strength, whereas we observe effects on $\sigma_{\mathrm{dc}}$ already for weak disorder. Instead, the key distinction is in the presence or absence of $p$ - $h$ symmetry. In order to explore this conjecture more fully, we have studied two other types of disorder: site disorder that preserves $p$ - $h$ symmetry and bond disorder that breaks $p$ - $h$ symmetry (by including next-nearest-neighbor hopping).

Particle-hole symmetric site disorder is introduced by adding random chemical potentials to the Hubbard model which couple with opposite sign to the density of up and down electrons, i.e., choose $\mu_{\mathbf{j}} \equiv \mu_{\mathbf{j} \sigma}=\sigma \mu_{\mathbf{j}}$ in (1). This type of disorder represents a random (Zeeman) magnetic field. For $U=0 p$ - $h$ symmetric site disorder has precisely the same effect as conventional site disorder, since moving in a given random chemical potential landscape or one obtained by reversing all the site energies is entirely equivalent. However, the behavior of the conductivity at finite $U$ is dramatically different. Figure 2 shows that $p$ - $h$ symmetric site disorder (with strength $\Delta_{\mu}^{\prime}$ ) has the same effect on $\sigma_{\mathrm{dc}}$ as bond disorder, i.e., conductivity decreases with increasing $\Delta_{\mu}^{\prime}$.

To seek final confirmation of our conjecture, we have also explored the effect of next-nearest-neighbor (nnn) hopping and randomness therein. Such longer ranging hybridization breaks $p$ - $h$ symmetry on a square lattice, since it connects sites on the same sublattice. We find that such disorder has the same effect as conventional site randomness, i.e., increases the conductivity at finite $T$. Thus in all four types of disorder, the behavior of the conductivity falls into the appropriate class based on the preservation or destruction of $p$ - $h$ symmetry, strengthening the evidence that it is this symmetry which is playing the crucial role in determining the effect of randomness on the transport properties.

We now turn to thermodynamic properties. The most direct measure of the Mott gap is from the compressibility, or from the behavior of density $\langle n\rangle$ as a function of chemical potential $\mu$, as shown in Fig. 3. The range of $\mu$ where $\langle n\rangle$ is constant (and the system is incompressible) is a direct measure of the gap in the spectrum. Hopping and $p$ - $h$-symmetric site disorder clearly stabilize the plateau of the density at half filling. On the other hand, conven-

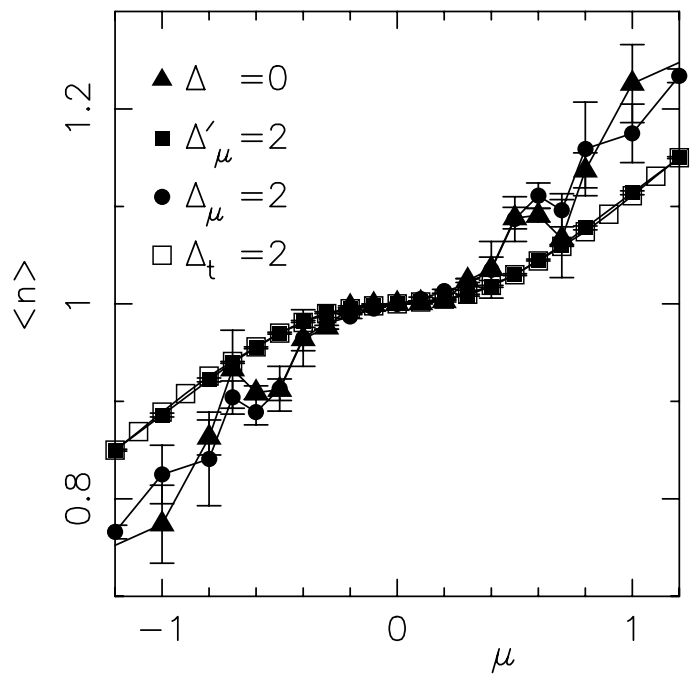

FIG. 3. The Mott gap is made more robust by the addition of bond disorder or particle-hole-symmetric site disorder (open and filled squares) of strength $\Delta=2 t=U / 2$, as indicated by the response of the density to changes in the chemical potential. For canonical site disorder (filled circle) the Mott gap is practically unaffected by this strength of randomness [19]. Calculations are for $T=t / 8=W / 64$ on a $8 \times 8$ lattice.

tional site disorder (with $\Delta_{\mu}=U / 2$ ) has a compressibility which is indistinguishable (within the computational possibilities) from the clean system.

The density of states (DOS) at the Fermi level $N(0)$ gives valuable information on the effect of disorder on the Mott gap. In the pure system, QMC studies have shown that the DOS exhibits a clear Mott gap with $N(0) \rightarrow 0$ as $T$ is lowered to zero. The nonzero values of $N(0)$ we obtain at nonzero $T$ reflect the small residual slopes in the plateaus in the $\langle n\rangle$ vs $\mu$ plot (cf. Fig. 3); at lower $T, N(0)$ approaches zero just as the plateaus become perfectly flat. The behavior of $N(0)$ at a fixed low $T$ as a function of the strength of the various types of disorder is given in Fig. 4. $N(0)$ is rather insensitive to $p$ - $h$ symmetric disorder $\left(\Delta_{t}\right.$ and $\left.\Delta_{\mu}^{\prime}\right)$ and is even reduced by it: the Mott gap persists. On the other hand, $p-h$ symmetry breaking disorder $\left(\Delta_{\mu}\right.$ and $\Delta_{t}^{\prime}$ ) clearly enhances $N(0)$, i.e., fills up the Mott gap.

Our results provide a clear numerical demonstration of the key role of particle-hole symmetry. The effects can also be understood qualitatively as follows: In the clean case, at $\langle n\rangle=1$ and strong coupling, the DOS consists of an occupied lower Hubbard band (LHB) and an unoccupied upper Hubbard band (UHB), separated by a charge gap of the order of $U$. In the case of $p-h$-symmetric disorder, the effect of disorder on LHB and UHB is identical. Therefore the Fermi energy remains in the middle of the gap: this enables the insulating behavior and Mott gap to stay intact. A stabilized charge gap for $p$ - $h$-symmetric site disorder is evident since double occupation is strongly suppressed. For $n n$-hopping disorder a simple argument is less obvious, but the data in Fig. 3 clearly show that these two cases fall into the same class. When $p-h$ symmetry is broken, the LHB and the UHB will be affected differently; 


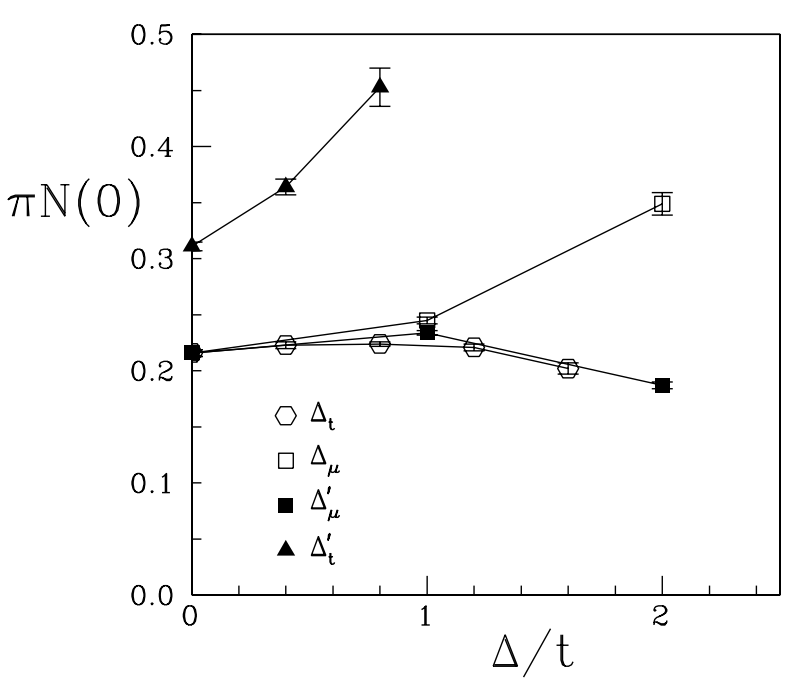

FIG. 4. Behavior of the density of states $\pi N(0)$ at the Fermi level and at fixed low temperature as a function of disorder strength $\Delta / t$ for various types of disorder. All data are for $T=t / 6$, except data for randomness in next-nearest-neighbor hopping (disorder strength $\Delta_{t}^{\prime}$ ) which are at temperature $T=$ $t / 5$ (the value $t^{\prime}=0$ is used) [19]. Other parameters are as in Figs. 1 and 2.

different numbers of states will appear at either side of the gap. As a consequence, the Fermi energy ends up in one of the tails of the DOS, resulting in an enhanced $N(0)$ (cf. Fig. 4) and increased conductivity (Fig. 2). The fact that the states introduced by disorder are localized [20] will keep the system in an insulating state (cf. Fig. 2).

Conclusions. - In this Letter, we have shown that $p$ - $h$ symmetry plays a decisive role in determining the effect of randomness on transport and thermodynamic properties of the half-filled Hubbard model. By exploring four different types of disorder, of which two preserve $p$ - $h$ symmetry and two break it, we demonstrate that a classification by this symmetry allows us to understand the effect of disorder on $\sigma_{\mathrm{dc}}(T)$, the charge gap, and the compressibility. The presence of $p$ - $h$ symmetry is found to have a protective influence on the charge gap.

A related example where symmetry plays a crucial role in the effects of disorder is the case of localization in the superconducting phase, where the quasiparticles are described by a Bogoliubov-de Gennes Hamiltonian [11]. In this case, one can classify the system according to the presence or absence of time reversal and spin rotation symmetries, and it is found in one dimension that in the absence of spin rotation symmetry, the conductance decays algebraically with system size, while in the symmetric case it decays exponentially. Therefore, in this situation as well, the extra spin rotation symmetry leads to a strengthening of insulating behavior.

The question of the behavior of the half-filled fermion Hubbard model as disorder is added is furthermore reminiscent of similar issues in the $p$ - $h$-symmetric boson Hubbard model [6]. At generic densities, it is believed that a new "Bose glass" phase arises to intervene in the original ground state phase diagram between superfluid and Mott insulating phases, but the situation at the $p$ - $h$-symmetric tip of the Mott lobe is uniquely different. Our work is a first step in the analysis of the nature of the behavior of the fermionic model.

The authors thank Piet Brouwer, Andreas Ludwig, and George Sawatzky for helpful discussions. The research of R. T. S. is supported by Grant No. NSF-DMR-9985978.

[1] E. Abrahams, S. V. Kravchenko, and M. P. Sarachik, Rev. Mod. Phys. 73, 251 (2001), and references therein.

[2] A. M. Finkel'stein, Zh. Eksp. Teor. Fiz. 84, 168 (1983) [Sov. Phys. JETP 57, 97 (1983)].

[3] P. J. H. Denteneer, R. T. Scalettar, and N. Trivedi, Phys. Rev. Lett. 83, 4610 (1999).

[4] R. N. Bhatt and P. A. Lee, Phys. Rev. Lett. 48, 344 (1982); M. A. Paalanen, S. Sachdev, R. N. Bhatt, and A. E. Ruckenstein, Phys. Rev. Lett. 57, 2061 (1986); M. A. Paalanen, J. E. Graebner, R. N. Bhatt, and S. Sachdev, Phys. Rev. Lett. 61, 597 (1988).

[5] A. M. Goldman and N. Markovic, Phys. Today 51, No. 11, 39 (1998).

[6] M. P. A. Fisher, P. B. Weichman, G. Grinstein, and D. S. Fisher, Phys. Rev. B 40, 546 (1989).

[7] G. Blatter et al., Rev. Mod. Phys. 66, 1125 (1994).

[8] F. J. Wegner, Z. Phys. B 35, 207 (1979); R. Gade and F. J. Wegner, Nucl. Phys. B360, 213 (1991).

[9] P.W. Brouwer, C. Mudry, and A. Furusaki, cond-mat/0009198.

[10] T. Senthil and M.P. A. Fisher, Phys. Rev. B 61, 9690 (2000).

[11] P. W. Brouwer, A. Furusaki, I. A. Gruzberg, and C. Mudry, Phys. Rev. Lett. 85, 1064 (2000), and references therein.

[12] S. R. White, D. J. Scalapino, R. L. Sugar, E. Y. Loh, J.E. Gubernatis, and R.T. Scalettar, Phys. Rev. B 40, 506 (1989).

[13] T. Vojta, F. Epperlein, and M. Schreiber, Phys. Rev. Lett. 81, 4212 (1998); X. Waintal, G. Benenti, and J.-L. Pichard, Europhys. Lett. 49, 466 (2000).

[14] D. L. Shepelyansky, Phys. Rev. Lett. 73, 2607 (1994).

[15] N. Trivedi, R. T. Scalettar, and M. Randeria, Phys. Rev. B 54, R3756 (1996). The formula for $\sigma_{\mathrm{dc}}$ is expected to hold when $T$ is lower than other energy scales in the problem (e.g., disorder).

[16] N. Trivedi and M. Randeria, Phys. Rev. Lett. 75, 312 (1995). The expression for $N(0)$ is valid at temperatures below characteristic frequency scales of structures in $N(\omega)$.

[17] M. Ulmke and R. T. Scalettar, Phys. Rev. B 55, 4149 (1997).

[18] We have verified that beyond a certain large site disorder $\left(\Delta_{\mu} \geq 4 t\right) \sigma_{\mathrm{dc}}$ decreases again.

[19] Particle-hole symmetric disorder has no sign problems at half-filling; data are better then, and simulations can go to lower $T$.

[20] Y. Otsuka, Y. Morita, and Y. Hatsugai, Phys. Rev. B 58, 15314 (1998). 